\title{
The Role of Networks in Racial Disparities in HIV Incidence Among Men Who Have Sex with Men in the United States
}

\author{
Stephen Bonett ${ }^{1}$ (i) $\cdot$ Steven Meanley ${ }^{1} \cdot$ Robin Stevens $^{1} \cdot$ Bridgette Brawner $^{1} \cdot$ José Bauermeister $^{1}$
}

Published online: 24 January 2020

(c) Springer Science+Business Media, LLC, part of Springer Nature 2020

\begin{abstract}
Network factors have been proposed as potential drivers of racial disparities in HIV among Black and Latino men who have sex with men (MSM). This review aimed to synthesize the extant literature on networks and racial disparities in HIV among MSM and identify potential directions for future research. We searched databases for peer-reviewed articles published between January 1, 2008 and July 1, 2018. Articles were included if the sample was comprised primarily of racial/ethnic minority MSM and measured one or more network characteristics. $(n=25)$. HIV prevalence in networks, social support, and structural barriers were linked to disparities in HIV for Black MSM. Future research should focus on intervention development around social support and other strategies for risk reduction within networks. Given the contribution of structural factors to racial/ethnic HIV disparities, network-level interventions should be paired with policies that improve access to housing, jobs, and education for MSM.
\end{abstract}

Keywords Racial disparities $\cdot$ Networks $\cdot$ Systematic review $\cdot$ HIV/AIDS $\cdot$ Men who have sex with men

\section{Resumen}

Los factores de redes han sido propuestos como posibles elementos contribuyentes a las disparidades raciales en el VIH entre hombres Afro-Americanos y Latinos que tienen sexo con hombres (HSH). Esta revisión sintetiza la literatura existente sobre redes y disparidades raciales en el VIH entre HSH e identifica posibles direcciones para investigaciones en el futuro. Revisamos estudios publicados entre el 1 de enero del 2008 y el 1 de julio, 2018. Los estudios se incluyeron si la muestra estuvo compuesta principalmente por HSH de minorías raciales/étnicas y si midieron una o más características de la red (n =25). La prevalencia del VIH en redes, el apoyo social, y las barreras estructurales estaban vinculadas a los disparidades en el VIH entre Afro-Americanos y Latinos HSH. Investigaciones en el futuro deberían centrarse en el desarrollo de intervenciones relacionadas con el apoyo social y otras estrategias para la reducción de riesgo dentro de redes. Dada la importancia de factores estructurales, las intervenciones de red deben estar emparejadas con políticas que mejoren el acceso a la vivienda, el empleo, y la educación para los HSH de minorías raciales/étnicas.

Men who have sex with men (MSM) continue to bear the largest burden of the HIV epidemic. In 2017 MSM in the United States (US) accounted for $82 \%$ of incident HIV cases among men [1]. Black MSM (BMSM) and Latino MSM experience disproportionately high rates of HIV, making up $37 \%$ and $29 \%$ of incident cases among MSM, respectively [1]. The disparity is even greater for young BMSM, ages

Stephen Bonett

stepdo@ nursing.upenn.edu

1 School of Nursing, University of Pennsylvania, 418 Curie Blvd, Suite 222L, Philadelphia, PA 19104, USA
13-24, who made up 51\% of the new HIV infections among young MSM in 2017 [1].

HIV prevention efforts have largely focused on reducing risk behaviors in populations affected by the disease. Research has shown, however, that these behavioral factors do not adequately explain racial disparities in HIV infection. A seminal paper from Millett et al. [2] found that BMSM report lower rates of substance abuse and fewer sexual partners than White MSM, and report similar rates of condomless anal intercourse (CAI), commercial sex work, sex with a partner who is known to be HIV positive, and lifetime HIV testing. A meta-analysis of HIV risk factors in MSM [3] found that BMSM were less likely to have a history of 
substance abuse and more likely to use condoms. Despite these risk-reducing behaviors, BMSM were found to have higher rates of HIV and were less likely to have initiated medical treatment. Importantly, this study found BMSM experienced structural barriers, including poverty, incarceration and unemployment, at twice the rate of White MSM. These findings suggest that behavioral factors are not a primary contributor to the racial disparities in HIV incidence in MSM, and that higher-level distal factors (e.g. network factors or structural factors) likely play a more prominent role [4].

One legacy of the Millett et al. [2] paper is the implication of sexual networks as a possible driver of racial disparities in HIV. This suggestion sparked a wave of inquiry into how the dynamics of social and sexual networks relate to HIV transmission risk [5-8]. It has been hypothesized that racial disparities in HIV are perpetuated and exacerbated by increased prevalence of HIV within Black communities and high racial homophily (i.e., the degree to which members of a network have the same racial identity) in the sexual networks of BMSM [9]. Social support within networks has also been identified as an influential factor in determining sexual risk behavior, viral load suppression, and HIV testing [10-12]. Recently, researchers have proposed network viral load, which refers to the total HIV viral activity within a connected network, as a metric of HIV risk [13].

The persistent racial disparities, particularly between BMSM and non-Hispanic White MSM, in HIV incidence represents an urgent public health issue. BMSM experience simultaneous marginalization related to racial identity and sexual identity and are disproportionately burdened by a highly stigmatized disease. Network factors could play an important role in understanding how racial disparities in HIV have developed and persisted. The present review aims to (1) collect and summarize the existing body of literature on networks and HIV risk and disparities, (2) place the findings on network factors in the context of research on individual and structural factors, (3) explore implications for intervention and policy development, and (4) propose directions for future research.

\section{Methods}

\section{Search Strategy}

In collaboration with an academic librarian and an established HIV researcher, we collected scientific literature relevant to network factors in HIV among MSM from four databases representing the most comprehensive collections of behavioral HIV research: Pubmed, Scopus, PsycINFO, and Sociological Abstracts. Search logic was constructed by combining terms related to networks, social support, HIV and other STIs, sexual risk behavior, racial disparities, and MSM (Fig. 1). The search was limited to peer-reviewed articles published between January 1, 2008 and July 1, 2018, representing all literature published since the release of the seminal review from Millett et al. in 2007 that identified network factors as possible drivers of racial disparities in HIV.

\section{Inclusion/Exclusion Criteria}

We included articles if they described an empiric study that: (1) quantitatively measured either the structure (i.e., size, density, concurrency) or function (i.e., social support) of a social, sexual, or drug network, (2) measured one or more relevant HIV outcomes (i.e., HIV prevalence, seroconversion, sexual risk behavior), (3) sampled primarily racial/ ethnic minority men, with more than $50 \%$ of the study population being racial/ethnic minorities, (4) sampled primarily MSM, with more than $50 \%$ of the study population being MSM, (5) took place in the US, (6) was published in a peer-reviewed journal, and (7) was published in English or Spanish. The first author screened the titles and abstracts of all articles identified from the search query using the inclusion and exclusion criteria and reviewed the full texts of the remaining articles in detail. Those marked for exclusion were annotated with specific reasons for exclusion. Any article that was not clearly a candidate for inclusion or exclusion was discussed with a second reviewer, and consensus was reached regarding inclusion/exclusion.

\section{Data Extraction}

Data were extracted from the reviewed articles and compiled into a table of evidence. For each included article, we extracted the goal of the study, the data source (site of recruitment for primary data, name of original study for secondary data), the setting, the sample size, demographic characteristics of the sample, type of network measured, HIV outcome measures, and key findings.

\section{Results}

A total of 1184 articles were found over the four databases queried. After removing duplicates, 656 unique articles remained for review. Of these, 581 were excluded in the title and abstract screening phase. An additional 50 articles were excluded in the full text screening phase: 22 studies were excluded because they did not include a network measure, 18 because they did not measure a relevant outcome, nine because they were not empiric papers, and one because it was not peer reviewed. The remaining 25 articles met criteria and were included in this review (Fig. 2). 
Fig. 1 Search logic used for Pubmed query, as an example

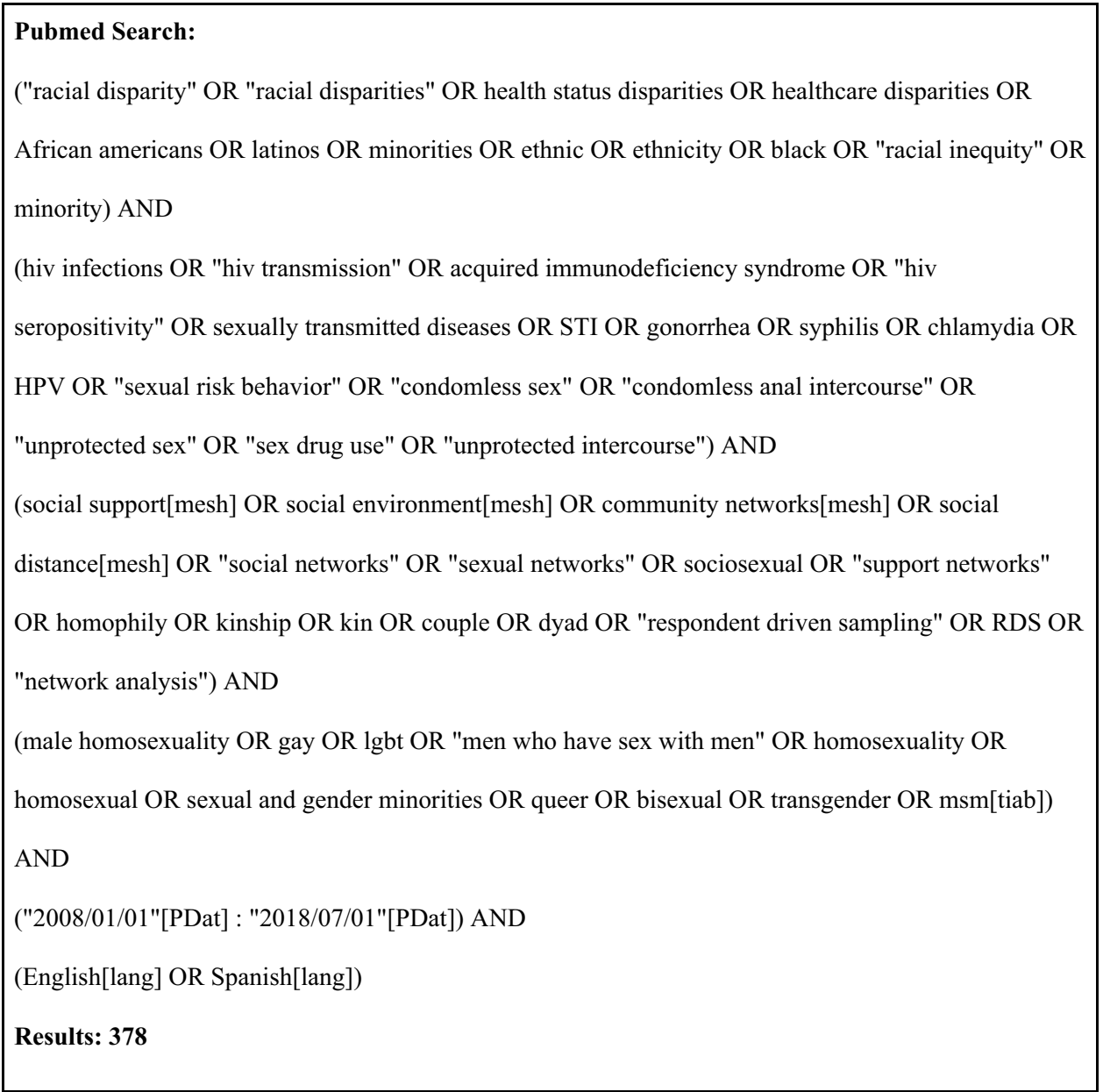

A summary of the data extracted for this review can be found in Table 1. Three of the included studies used a prospective cohort design, while the remaining studies used a cross-sectional design $(n=22)$. All but one of the included studies $(n=24)$ sampled from large and medium metro areas, as defined by the 2013 NCHS urban-rural classification scheme [14]. One study sampled 13 distinct communities and included several small metro areas and neighborhoods in large central cities [15]. Seven studies focused on young MSM, and the remaining studies included adult or older MSM $(n=18)$. Sample sizes in the studies ranged from 121 to 10,295 . Twelve studies enrolled only BMSM and Black transwomen. Nine studies measured social networks only, six measured sexual networks only, and ten studies measured both sexual and social networks. Of the studies reviewed, 22 measured egocentric networks (i.e. information is collected only from the participant) and three measured sociometric networks (i.e. information is obtained both from participants and from their network members). HIV outcomes measured included sexual risk behaviors, HIV prevalence, and seroconversion during study period.

\section{Structural Network Determinants}

\section{Size and Density}

The size and density of both social and sexual networks are possible predictors of HIV risk. Three studies found that the size of one's social network had an effect on sexual risk behavior, although the effect was different for BMSM than for Latino MSM. Larger social networks were associated with increased CAI in BMSM, while smaller social networks were associated with increased CAI in Latino MSM [16-18]. This difference by race was seen both in older MSM and young MSM (YMSM) and was consistent across studies in various US cities. In one study it was found that greater density amongst members of a social network was associated with lower rates of serodiscordant condomless intercourse (SDCI) with a non-primary partner, an effect that did not differ by race/ethnicity [19]. Another study found no connection between social network density and sexual risk behavior [20]. 
Fig. 2 PRISMA flow diagram for systematic review. From Ref. Moher et al. [53]
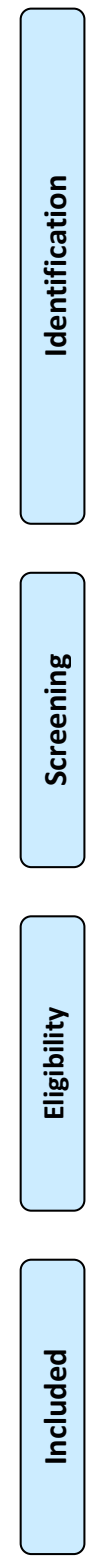

The studies that focused on size and density of sexual networks were not conclusive. One large multi-city study of BMSM found that larger sexual networks had a bivariate association with higher rates of CAI [21], however a separate analysis of the same dataset that used SDCI as an outcome rather than any CAI found no association between sexual network size or density and SDCI in multivariate models [22]. Similarly, for YMSM in Chicago, no association was found between size or density of sexual networks and CAI [23]. Structural characteristics of social and sexual networks are inconsistent predictors of sexual risk behavior. The structure of social networks is more salient to behavior than the structure of sexual networks,
Records identified through

database searching:

Pubmed

( $n=378)$

Scopus

$(n=449)$

Sociological Abstracts

( $n=46)$

PsychINFO

$(n=311)$

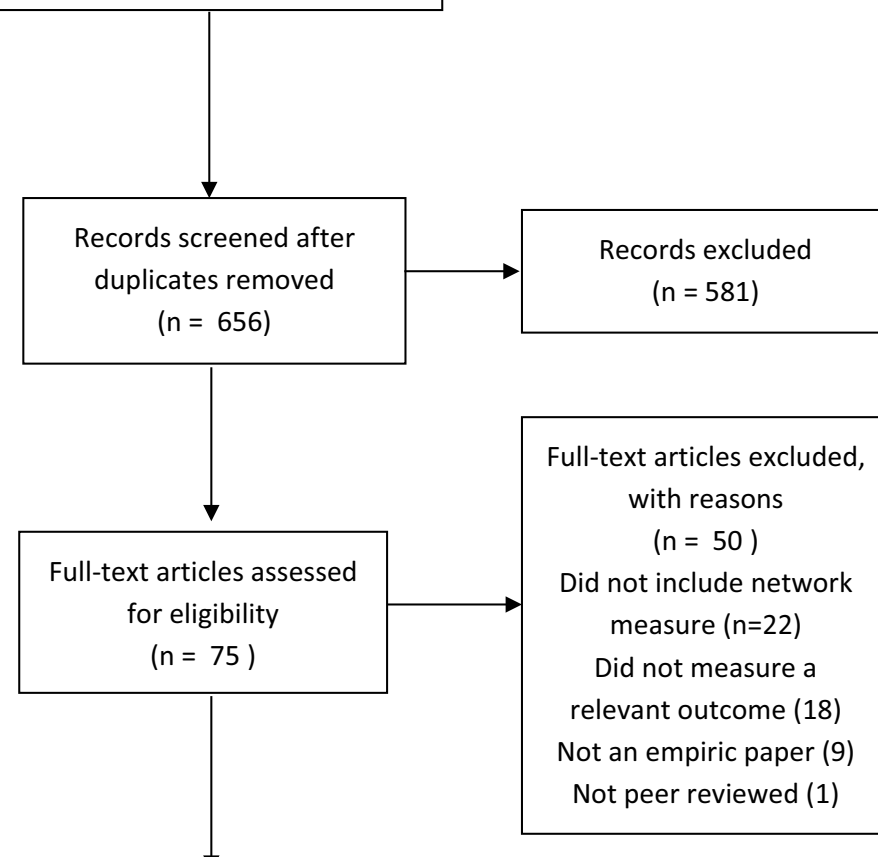

Studies included in review

$(n=25)$ and network factors manifest differently across racial/ethnic groups.

\section{Functional Network Determinants}

\section{Social Support}

The role of support networks and social support was investigated in seven of the reviewed studies. Studies varied in how they measured social support, although most either measured general social support, or measured specific subdomains of social support (i.e. emotional, instrumental, informational, appraisal) [24]. Two studies included measures of "HIV-related" social support [15, 20]. Most studies 


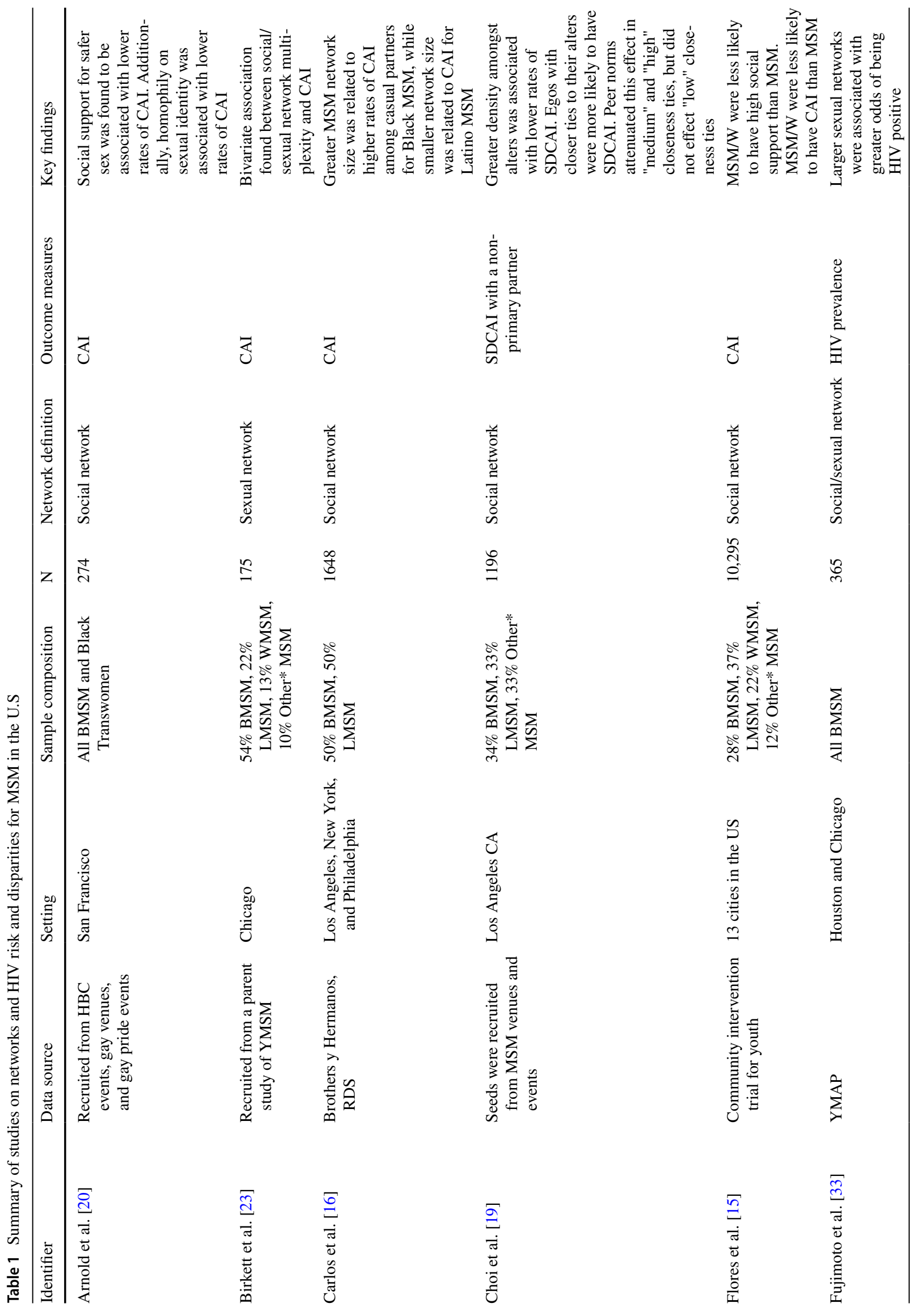




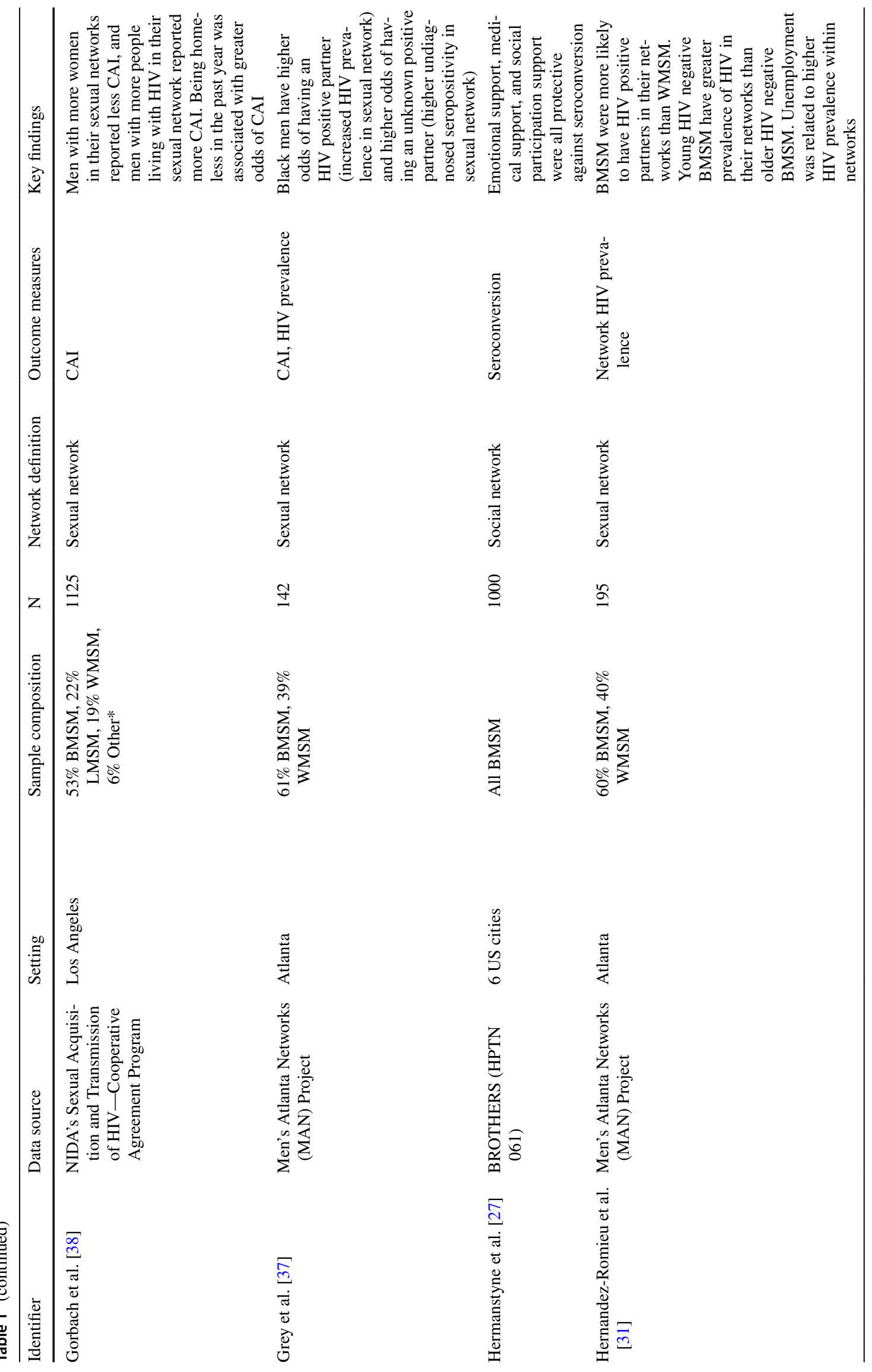




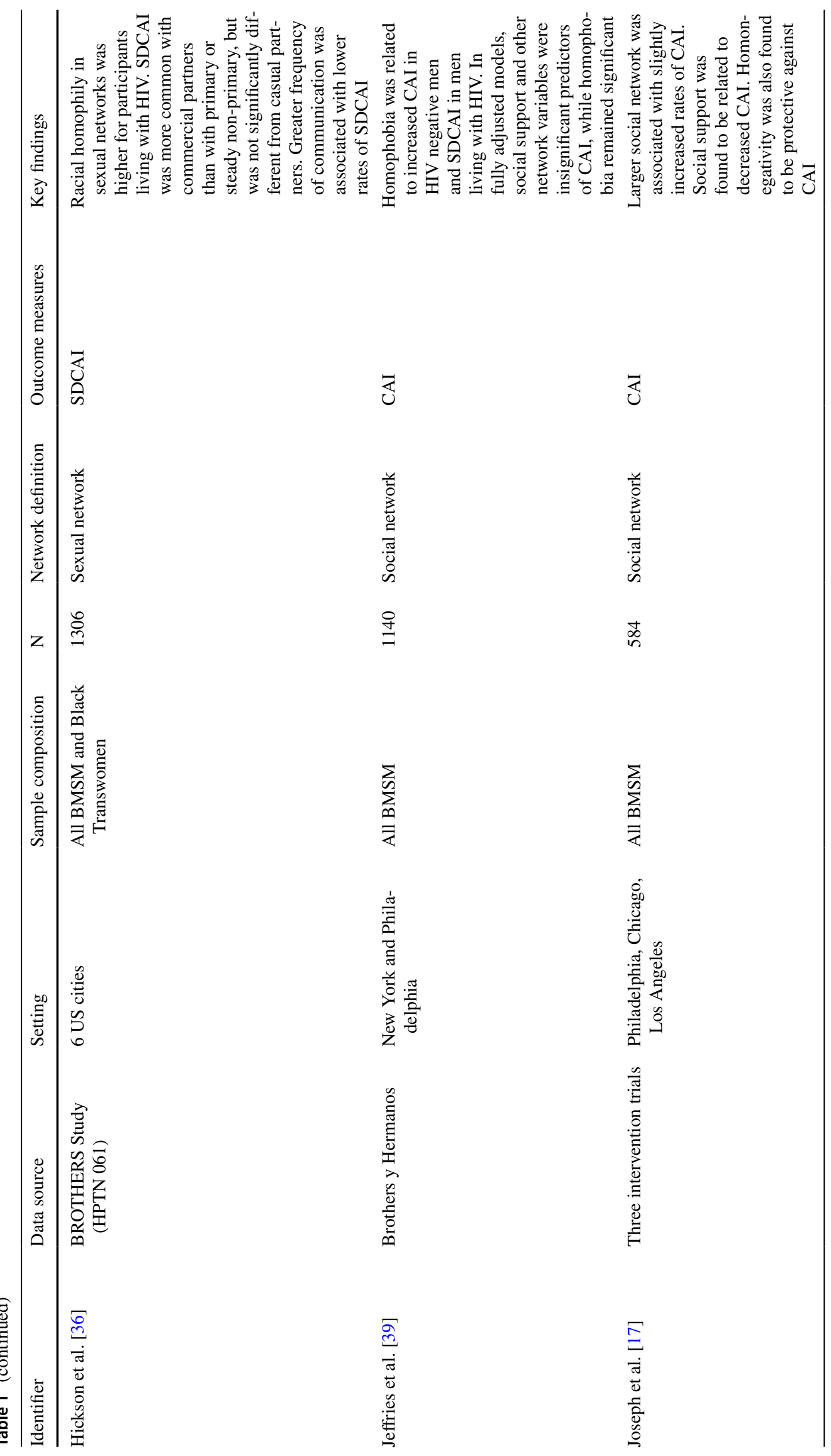




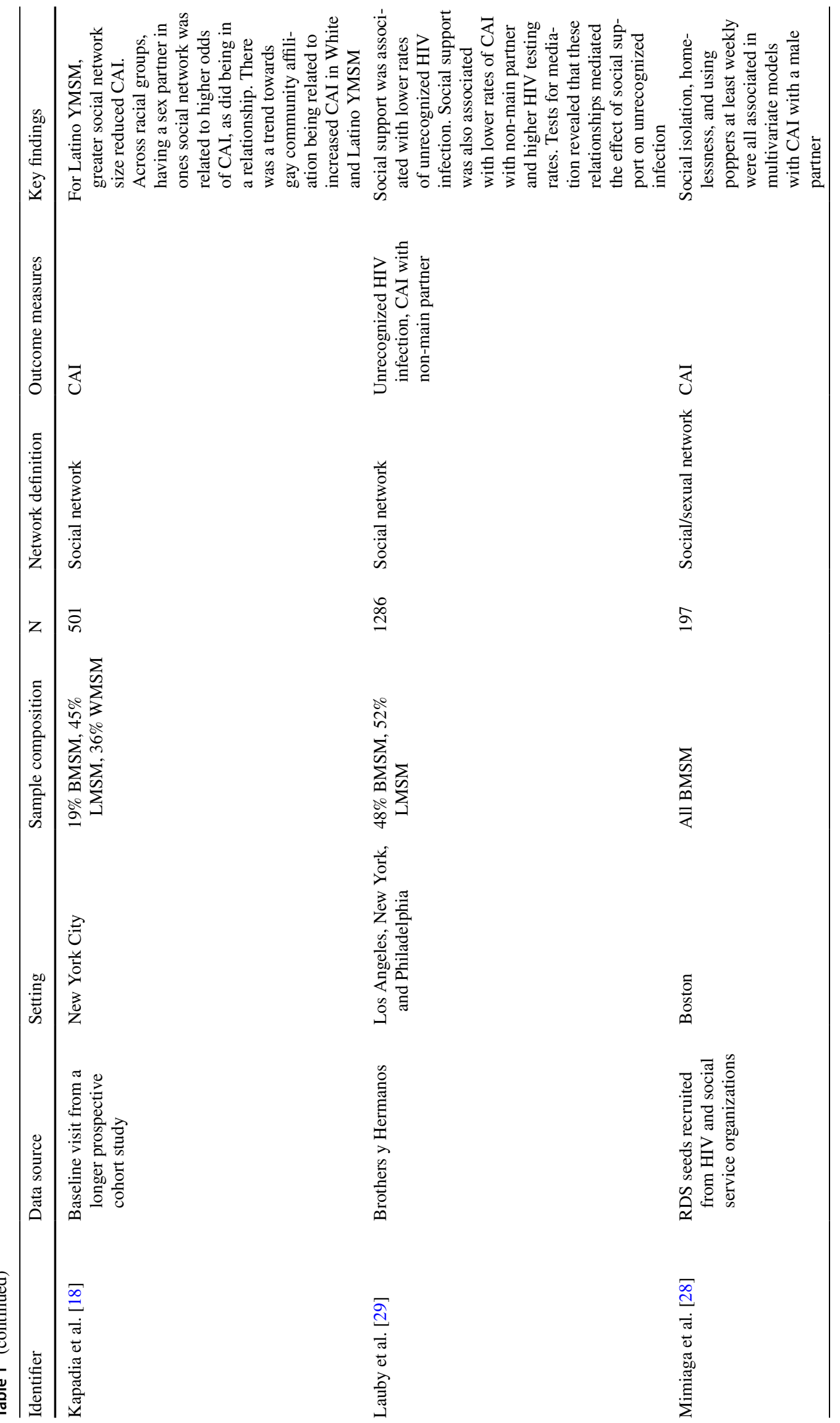




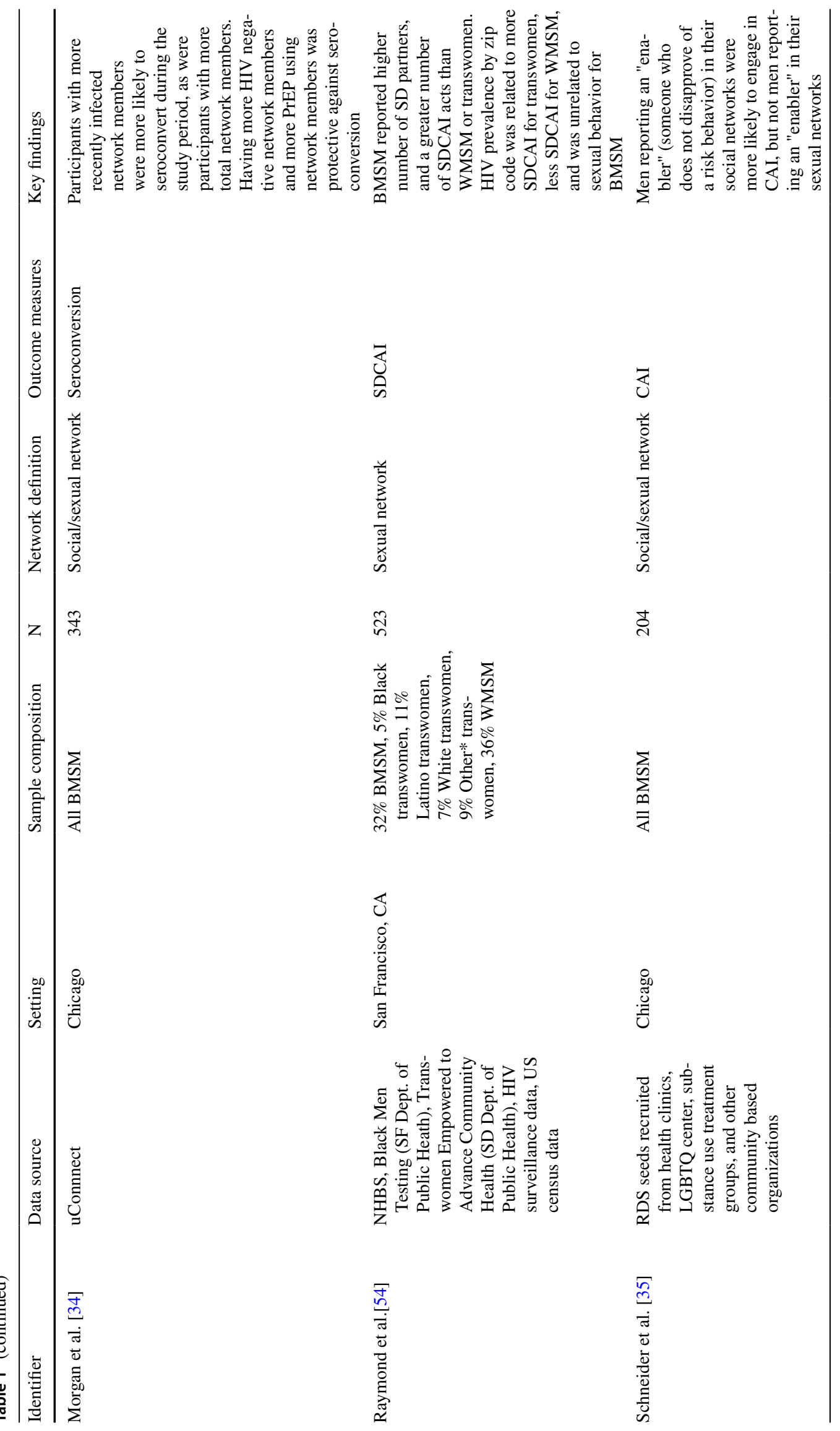




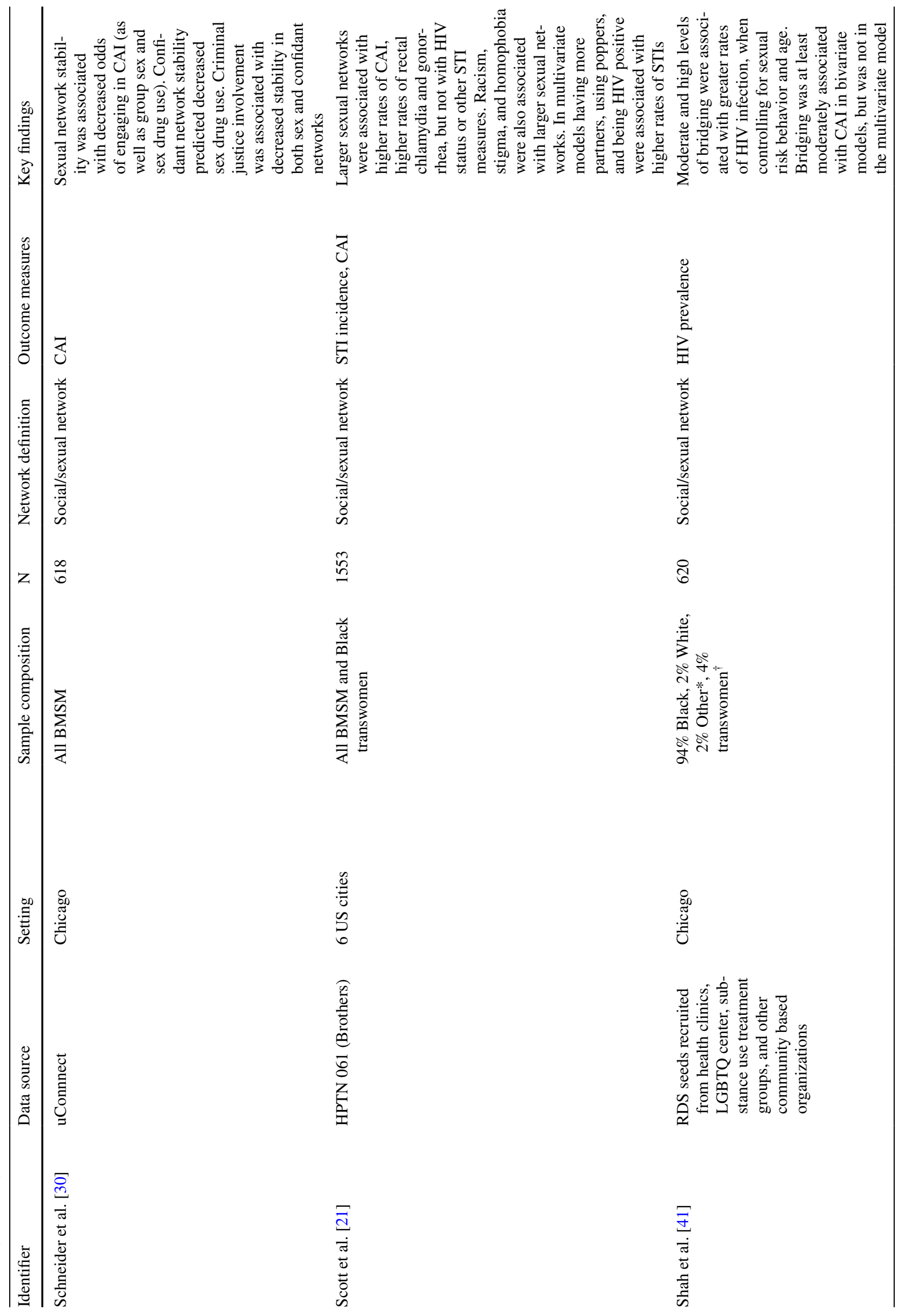




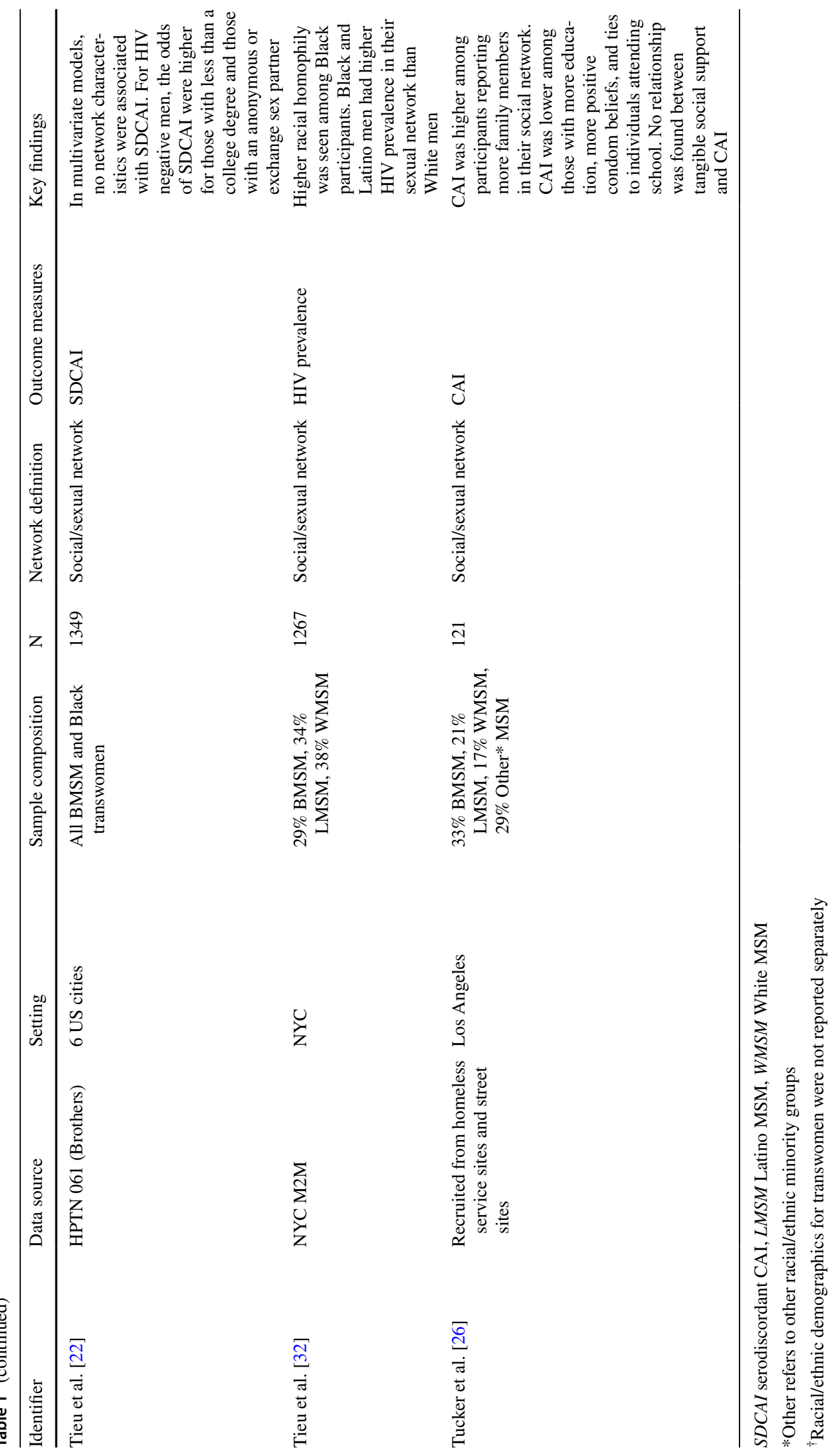


measured perceived social support (i.e. a personal belief that social support is available and adequate) [25], although one study measured enacted social support [26]. The majority of the studies measured network-wide social support, but three studies used an actor-specific measure of social support where support from individual network members was assessed [20, 26, 27]. General perceived social support within a network was associated with lower rates of CAI [17, $28,29]$ as was a measure of actor-specific HIV-related social support [20]. Lauby et al. [29] found that the relationship between general perceived social support within a network and HIV infection in their study was partially explained by changes in CAI. The direct impact of perceived social support on HIV incidence was explored in one notable prospective cohort study of BMSM in six major US cities, which found that having more people in one's emotional support network, medical support network, and social participation support network were all protective against seroconversion during the study period [27].

Two studies found no connection between social support and sexual risk behavior. One small study of homeless youth found no connection between actor-specific tangible enacted social support and sexual risk behavior [26]. A large multicity study of YMSM found that social support was unrelated to CAI [15], although this study employed a measure of HIV-related social support within a network that had not previously been used or validated. Social support is an important determinant of sexual risk behavior and HIV risk, although differences in measurement and study population might modify this relationship.

\section{Compositional Network Determinants}

\section{Stability}

To our knowledge, only one study has investigated the effects of network stability (i.e., the extent to which the members of an individual's network stay constant over time) on sexual risk behavior [30]. This study found that stability in sexual networks was associated with decreased odds of engaging in condomless sex, as well as with decreases in other sexual risk behaviors. Stability in social networks was found to be protective against sex drug use, but was not protective against condomless sex. Network stability remains a potentially important factor in racial disparities in HIV and additional research is warranted.

\section{Racial Homophily}

Four studies explored aspects of the hypothesis that racial disparities in HIV are amplified by high HIV prevalence among BMSM and high racial homophily in the sexual networks of BMSM. The networks of BMSM in Atlanta had higher HIV prevalence than those of White MSM [31]. BMSM in New York City had higher rates of HIV and higher levels of racial homophily when compared to White MSM [32]. Birkett et al. [23] found that racial homophily in egocentric networks was associated with participant HIV status among BMSM, but not among other racial ethnic groups. One notable exception to this pattern of findings was a multi-city study with young BMSM that observed no connection between egocentric network HIV prevalence and participant HIV status [33].

\section{Network Viral Load}

Morgan et al. [34] investigated the effects that differences in HIV transmissibility among network members plays in HIV risk, a concept known as network viral load. This prospective cohort study exploring network factors and HIV found that for young BMSM in Chicago, each additional recently infected HIV positive partner (high transmissibility) in one's network was associated with 13 times greater odds of seroconversion during the study period. There was no relationship between number of long-term infected partners (low transmissibility) and seroconversion, and number of HIV negative partners and number of partners using PrEP (both have negligible transmissibility) were each significant protective factors against seroconversion. These results are consistent with network viral load being a key HIV risk factor.

\section{Non-Network Correlates}

In the reviewed literature, constructs at multiple ecological levels were implicated consistently across many studies. A majority of the studies reviewed $(n=18)$ examined factors related to HIV risk and disparities at multiple levels in additional to network factors; the findings related to these non-network factors are summarized here.

Nine studies reported results related to psychosocial factors including six that investigated how attitudes and norms influence sexual risk behavior and three that explored the role that communication within relationships plays in HIV risk. Norms and attitudes were found to be consistently connected to sexual risk behavior [15, 16, 19, 20, 26, 35], and partner communication and power dynamics emerged as key relationship factors that could influence HIV risk and disparities [22, 36, 37].

Nine studies included structural factors in their main analysis. Socioeconomic status was the most commonly measured structural factor $(\mathrm{n}=5)$, but a small number of studies explored incarceration $(n=2)$ or discrimination $(n=2)$ as a determinant of HIV risk. Higher educational achievement, current employment, and stable housing were all found to be related to lower HIV risk [22, 28, 31]. Incarceration was related to participation in exchange sex and instability in 
social and sexual networks, but was not tied directly to HIV outcomes [30, 38]. There was not clear consensus regarding the effects of discrimination, either related to racism or to homophobia, on HIV risk and additional research in this area is warranted $[21,39]$.

\section{Discussion}

We sought to conduct a systematic review of the literature on networks and racial disparities in HIV to describe the state of the science and identify potential directions for future research. The findings suggest that size and density of sexual networks may be less influential than the size and density of social networks, that social support plays a protective role regarding HIV risk, and that racially homophilous networks with high HIV prevalence are linked to disparities in HIV. In addition to network factors, psychosocial factors and structural factors were also found to be involved in determining HIV risk and were often studied alongside network factors. This review highlights the importance of studying network characteristics in understanding racial disparities in the HIV epidemic. Where individual behavioral factors fail to explain disparities, network factors and their antecedent structural factors emerge as key drivers of sexual risk behavior and racial disparities in HIV incidence.

Networks have emerged as a central factor in HIV risk and disparities. While a great deal of research has measured structural characteristics of networks such as size and density, the relationship between these structural network factors and HIV risk and disparities is not clear. Little evidence was found to suggest the structure of sexual networks was highly predictive of HIV risk, and the relationship between the structure of social networks and risk was complicated. Of note, we discovered that social network size had different effects on CAI in BMSM and Latino MSM samples. We believe this may be attributed to the different relationships that Black and Latino men might have to the mainstream gay community. Patterns of racism in the MSM community, including racialized sexual stereotypes [40], may lead to Latino MSM finding greater support within this community, while BMSM may experience alienation or discrimination. Thus, as we consider the role of social networks in HIV risk among MSM, it is important to acknowledge varying effects that may be experienced among racial/ethnic minority groups. While basic structural characteristics such as size and density should still be measured, future research that measures more complex measures of network position, such as bridging [41], could offer additional insight into how networks affect HIV risk.

Social support within networks was well studied in the literature surrounding HIV risk and disparities. Social support was seen to be protective against HIV infection among
BMSM. Additionally, there was evidence that this relationship was partially mediated by the effect of social support on sexual risk behavior. One of the few prospective cohort studies reviewed found a direct relationship between social support and decreased seroconversion [34]. These findings are compelling because they reveal the influence of social support on HIV risk, as well as suggesting sexual risk behavior as a potential mechanism by which this effect operates. Several evidence-based interventions for racial/ ethnic minority MSM have been described that leverage social networks to influence behavior change (e.g. Many Men, Many Voices and d-up: Defend Yourself! [42, 43]), but little is known about the effects of these interventions at the network level. Research and intervention development should focus on evaluating their impact on network-level characteristics (e.g., changes in size, density, heterogeneity) and its mechanisms of change (e.g., increased availability of social support), alongside HIV outcomes, in an effort to inform strategies that address racial disparities in HIV.

The compositional characteristics of networks offered important insights into the connection between networks and HIV disparities. The evidence largely supported the hypothesis [7] that BMSM tend to have racially homophilous networks and networks with greater HIV prevalence than White MSM. It was found that viral load within a network emerged as a highly predictive correlate of seroconversion. This suggests that network viral load may be a more salient measure of HIV risk within a network than network HIV prevalence. Assessing network viral load also has several advantages over community viral load, another common measurement of aggregated viral activity [44]. Community viral load captures transmissibility within a geographic area. Since sexual networks often span wide geographic areas, network viral load offers a more direct measurement of transmissibility within socially defined networks.

While compositional characteristics of networks are an important factor in HIV risk and disparities, addressing network HIV prevalence and racial homophily through policy and intervention could prove problematic. The elevated rates of HIV within the networks of BMSM are both the cause and the consequence of racial disparities, and offers little direction for intervention. In fact, seeking to address racial homophily without mitigating the structural forces that have historically shaped these networks (e.g. racism, segregation) will be insufficient $[45,46]$. Additionally, focusing on racial homophily as a risk factor for HIV risks pathologizing love and connection among communities of color [47]. Intervention and policy should instead focus on testing and treatment, as well as improving access and adherence to PrEP and routine HIV care, to reduce viral load within the networks of BMSM. Increasing regular testing and improving partner communication skills could also help to narrow the knowledge gap about partner HIV status. 
Confirming a large body of research connecting individual level factors to HIV risk, psychosocial processes were found to have effects on sexual risk behavior. Theorydriven measurements should be incorporated into intervention design to better target the most salient psychological pathways leading to behavior change. Interventions that are theoretically based have been proven to reduce HIV risk behaviors and lower HIV incidence among MSM, including racial/ethnic minority MSM [48, 49].

Structural factors are key determinants of racial disparities in HIV. The mechanisms by which structural factors affect HIV disparities are not well understood and warrant investigation. The role of networks as mediators between structural factors and HIV disparities have largely been unstudied [50]. Research that measures how network stability might mediate a relationship between incarceration and HIV risk behavior and disparities would help illuminate this possible connection. Additionally, investigations into how structural discrimination such as homophobia and racism might manifest in social and sexual networks could be a fruitful direction for further research. The two studies in this review that explored the effects of discrimination used validated measures of perceived discrimination [21, 39], and future research should adopt or adapt these measures to insure comparability across studies.

Change in structural factors remains the foundation of an effective response to the persistent racial disparities in the HIV epidemic. Studies included in this review demonstrated that incarceration affects network stability and contributes to the interplay among sexual risk behaviors, sex work, and drug use. A limited body of research suggests that the criminal justice system could be a point of intervention to reduce these disparities [4]. Reforming policies and policing tactics that target sex workers, injection drugs users, and people of color could help to reduce network instability and HIV transmission [51]. Standardizing post-release support, including connection to testing and treatment, may serve to mitigate the effects of incarceration on sexual networks. Finally, policies that improve access to housing, jobs, and education for racial/ethnic minority MSM will be fundamental to efforts to reduce racial disparities in HIV.

\section{Limitations}

This review had several limitations. First, by limiting our review to studies that used quantitative measurement of network factors, we may miss important insights from foundational qualitative work. Second, our review was limited in scope. We only examined research that had an explicit focus on racial/ethnic disparities with individual-level outcomes in order to address our research question efficiently. As a result, our review excludes some network research (e.g., stochastic network modeling studies; see Fujimoto et al. for exemplar study) [52] that informs racial/ethnic HIV disparities. Additionally, the majority of the reviewed studies employed a cross-sectional study design, limiting our ability to draw conclusions about causation. Finally, there are inherent limitations to the egocentric (information derived solely from participant) network designs used by most studies in this review. Sociometric designs (information derived from all members of network), while more costly and burdensome, offer a more robust measurement of social and sexual networks. Future research should aim to measure complete networks over time in order to better understand how the dynamics of social and sexual networks effect HIV risk and disparities.

\section{Conclusions}

Racial disparities in HIV remain a pressing public health issue and a top priority to be addressed by future research, intervention development, and policy change. The existing literature supports a multilevel model of HIV risk that implicates factors at the individual, network, and structural levels in creating and propagating racial disparities. Structural, functional, and compositional characteristics of networks have all been linked to HIV risk behaviors or racial disparities, suggesting that networks play a key role in shaping these outcomes. Development of intervention and policy should look beyond individual level behavior change. Research and intervention that focuses on factors at the network and structural levels to effectively address racial disparities in HIV are needed. These higher-level factors, which require cultural and economic shifts, may address the fundamental causes of these disparities and hold the potential to affect lasting change.

Acknowledgements Support for this project was provided by the Rita and Alex Hillman Foundation and Hillman Scholars Program in Nursing Innovation (predoctoral fellowship). We sincerely thank Barbara Riegel, PhD RN FAHA FAAN for her contribution to reviewing drafts of this manuscript. We also thank Melanie Cedrone, MS and Jacqueline Bannon, $\mathrm{PhD} \mathrm{RN}$ for their advice and guidance on the process of systematic literature review.

Author Contributions SB conceived and designed the analysis, performed the literature search, screened the resulting literature for inclusion, extracted the data, appraised the quality of included studies, contributed to the synthesis and analysis of the results, and wrote the manuscript. SM appraised the quality of the included studies, contributed to the synthesis and analysis of the results, and reviewed and edited the manuscript. RS contributed to the synthesis and analysis of the results, and reviewed and edited the manuscript. BB contributed to the synthesis and analysis of the results, and reviewed and edited the manuscript. JB screened manuscripts that were ambiguous for inclusion, contributed to the synthesis and analysis of the results, reviewed and edited the manuscript, and provided mentorship to the first author throughout the manuscript development process. 


\section{Compliance with Ethical Standards}

Conflict of interest None of the authors are aware of any potential conflicts of interests regarding the findings of this study.

\section{References}

1. Centers for Disease Control and Prevention. HIV Surveillance Report, 2017. Vol 29. [Internet]. 2018. https://www.cdc.gov/hiv/ library/reports/hiv-surveillance.html. Accessed 21 Nov 2019

2. Millett GA, Flores SA, Peterson JL, Bakeman R. Explaining disparities in HIV infection among black and white men who have sex with men: a meta-analysis of HIV risk behaviors. AIDS. 2007;21(15):2083-91.

3. Millett GA, Peterson JL, Flores SA, Hart TA, Jeffries WL, Wilson $\mathrm{PA}$, et al. Comparisons of disparities and risks of HIV infection in black and other men who have sex with men in Canada, UK, and USA: a meta-analysis. Lancet. 2012;380(9839):341-8.

4. Maulsby C, Millett G, Lindsey K, Kelley R, Johnson K, Montoya D, et al. HIV among Black men who have sex with men (MSM) in the United States: a review of the literature. AIDS Behav. 2014;18(1):10-25.

5. Friedman SR, Bolyard M, Khan M, Maslow C, Sandoval M, Mateu-Gelabert P, et al. Group sex events and HIV/STI risk in an urban network. J Acquir Immune Defic Syndr. 2008;49(4):440.

6. Newcomb ME, Mustanski B. Racial differences in same-race partnering and the effects of sexual partnership characteristics on HIV risk in MSM: a prospective sexual diary study. J Acquir Immune Defic Syndr. 2013;62(3):329.

7. Raymond HF, McFarland W. Racial mixing and HIV risk among men who have sex with men. AIDS Behav. 2009;13(4):630-7.

8. Rosenberg ES, Khosropour CM, Sullivan PS. High prevalence of sexual concurrency and concurrent unprotected anal intercourse across racial/ethnic groups among a national, web-based study of men who have sex with men in the United States. Sex Transm Dis. 2012;39(10):741.

9. Amirkhanian Y. Social networks, sexual networks and HIV risk in men who have sex with men. Curr HIV/AIDS Rep. 2014;11(1):81-92.

10. Ayala G, Bingham T, Kim J, Wheeler DP, Millett GA. Modeling the impact of social discrimination and financial hardship on the sexual risk of HIV among Latino and Black men who have sex with men. Am J Public Health. 2012;102(2):242-9.

11. Friedman MR, Coulter RW, Silvestre AJ, Stall R, Teplin L, Shoptaw $\mathrm{S}$, et al. Someone to count on: social support as an effect modifier of viral load suppression in a prospective cohort study. AIDS Care. 2017;29(4):469-80.

12. Mashburn AJ, Peterson JL, Bakeman R, Miller RL, Clark LF. Influences on HIV testing among young African-American men who have sex with men and the moderating effect of the geographic setting. J Community Psychol. 2004;32(1):45-60.

13. Skaathun B, Khanna AS, Morgan E, Friedman SR, Schneider JA. Network viral load: a critical metric for HIV elimination. J Acquir Immune Defic Syndr. 2018;77(2):167-74.

14. Ingram DD, Franco SJ. NCHS urban-rural classification scheme for counties. Vital Health Stat. 2012;154:1-65.

15. Flores SA, Bakeman R, Millett GA, Peterson JL. HIV risk among bisexually and homosexually active racially diverse young men. Sex Transm Dis. 2009;36(5):325-9.

16. Carlos JA, Bingham TA, Stueve A, Lauby J, Ayala G, Millett GA, et al. The role of peer support on condom use among Black and Latino MSM in three urban areas. AIDS Educ Prev. 2010;22(5):430-44.
17. Joseph HA, Pan Y, Mendoza M, Harawa NT, Lauby J, Hosek SG, et al. HIV acquisition and transmission potential among African American men who have sex with men and women in three US cities. Arch Sex Behav. 2018;47(1):183-94.

18. Kapadia F, Siconolfi DE, Barton S, Olivieri B, Lombardo L, Halkitis PN. Social support network characteristics and sexual risk taking among a racially/ethnically diverse sample of young, urban men who have sex with men. AIDS Behav. 2013;17(5):1819-28.

19. Choi K-H, Ayala G, Paul J, Boylan R, Gregorich S. Social network characteristics and HIV risk among African American, Asian/ Pacific Islander, and Latino men who have sex with men. J Acquir Immune Defic Syndr. 2013;64(5):496-501.

20. Arnold EA, Sterrett-Hong E, Jonas A, Pollack LM. Social networks and social support among ball-attending African American men who have sex with men and transgender women are associated with HIV-related outcomes. Glob Public Health. 2018;13(2):144-58.

21. Scott HM, Irvin R, Wilton L, Van Tieu H, Watson C, Magnus $\mathrm{M}$, et al. Sexual behavior and network characteristics and their association with bacterial sexually transmitted infections among black men who have sex with men in the United States. PLoS ONE. 2015;10(12):0146025.

22. Tieu HV, Liu TY, Hussen S, Connor M, Wang L, Buchbinder S, et al. Sexual networks and HIV risk among black men who have sex with men in 6 US cities. PLoS ONE. 2015;10(8):0134085.

23. Birkett M, Kuhns LM, Latkin C, Muth S, Mustanski B. The sexual networks of racially diverse young men who have sex with men. Arch Sex Behav. 2015;44(7):1787-97.

24. Heaney CA, Israel BA. Social networks and social support. Health Behav Health Educ Theory Res Pract. 2008;4:189-21010.

25. Barrera M Jr. Distinctions between social support concepts, measures, and models. Am J Community Psychol. 1986;14(4):413-45.

26. Tucker JS, Hu J, Golinelli D, Kennedy DP, Green HD, Wenzel SL. Social network and individual correlates of sexual risk behavior among homeless young men who have sex with men. J Adolesc Health. 2012;51(4):386-92.

27. Hermansytne KA, Green HD, Cook R, Tieu HV, Dryer TV, Hucks-Ortiz C, et al. Social network support and decreased risk of seroconversion in black MSM: results of the BROTHERS (HPTN 061) Study. JAIDS J Acquir Immune Defic Syndr. 2018;78(2):163-8.

28. Mimiaga MJ, Reisner SL, Cranston K, Isenberg D, Bright D, Daffin $\mathrm{G}$, et al. Sexual mixing patterns and partner characteristics of black MSM in Massachusetts at increased risk for HIV infection and transmission. J Urban Health. 2009;86(4):602-23.

29. Lauby JL, Marks G, Bingham T, Liu KL, Liau A, Stueve A, et al. Having supportive social relationships is associated with reduced risk of unrecognized HIV infection among black and Latino men who have sex with men. AIDS Behav. 2012;16(3):508-15.

30. Schneider J, Cornwell B, Jonas A, Lancki N, Behler R, Skaathun B, et al. Network dynamics of HIV risk and prevention in a population-based cohort of young Black men who have sex with men. Netw Sci. 2017;5(3):381-409.

31. Hernández-Romieu AC, Sullivan PS, Rothenberg R, Grey J, Luisi $\mathrm{N}$, Kelley $\mathrm{CF}$, et al. Heterogeneity of HIV prevalence among the sexual networks of Black and White MSM in Atlanta: illuminating a mechanism for increased HIV risk for young Black MSM. Sex Transm Dis. 2015;42(9):505.

32. Tieu HV, Nandi V, Hoover DR, Lucy D, Stewart K, Frye V, et al. Do sexual networks of men who have sex with men in New York City differ by race/ethnicity? AIDS Patient Care STDs. 2016;30(1):39-47.

33. Fujimoto K, Flash CA, Kuhns LM, Kim JY, Schneider JA. Social networks as drivers of syphilis and HIV infection among young men who have sex with men. Sex Transm Infect. 2018;94(5):365-71. 
34. Morgan E, Skaathun B, Duvoisin R, Michaels S, Schneider JA. Are hiv seroconversions among young men who have sex with men associated with social network proximity to recently or longterm hiv-infected individuals? JAIDS J Acquir Immune Defic Syndr. 2018;77(2):128-34.

35. Schneider J, Cornwell B, Ostrow D, Michaels S, Schumm P, Laumann E, et al. Network mixing and network influences most linked to HIV infection and risk behavior in the HIV epidemic among black men who have sex with men. Am J Public Health. 2013;103(1):28-36.

36. Hickson DA, Mena LA, Wilton L, Tieu HV, Koblin BA, Cummings $\mathrm{V}$, et al. Sexual networks, dyadic characteristics, and HIV acquisition and transmission behaviors among Black men who have sex with men in 6 US cities. Am J Epidemiol. 2017;185(9):786-800.

37. Grey JA, Rothenberg R, Sullivan PS, Rosenberg ES. Racial differences in the accuracy of perceived partner HIV status among men who have sex with men (MSM) in Atlanta, Georgia. J Int Assoc Provid AIDS Care JIAPAC. 2015;14(1):26-322.

38. Gorbach PM, Murphy R, Weiss RE, Hucks-Ortiz C, Shoptaw S. Bridging sexual boundaries: men who have sex with men and women in a street-based sample in Los Angeles. J Urban Health. 2009;86(1):63-766

39. Jeffries WL, Marks G, Lauby J, Murrill CS, Millett GA. Homophobia is associated with sexual behavior that increases risk of acquiring and transmitting HIV infection among black men who have sex with men. AIDS Behav. 2013;17(4):1442-533.

40. Wilson PA, Valera P, Ventuneac A, Balan I, Rowe M, CarballoDiéguez A. Race-based sexual stereotyping and sexual partnering among men who use the internet to identify other men for bareback sex. J Sex Res. 2009;46(5):399-413.

41. Shah NS, Iveniuk J, Muth SQ, Michaels S, Jose JA, Laumann EO, et al. Structural bridging network position is associated with HIV status in a younger Black men who have sex with men epidemic. AIDS Behav. 2014;18(2):335-45.

42. Wilton L, Herbst JH, Coury-Doniger P, Painter TM, English G, Alvarez ME, et al. Efficacy of an HIV/STI prevention intervention for black men who have sex with men: findings from the manymen, manyvoices (3MV) project. AIDS Behav. 2009;13(3):532-44.

43. Jones KT, Gray P, Whiteside YO, Wang T, Bost D, Dunbar E, et al. Evaluation of an HIV prevention intervention adapted for Black men who have sex with men. Am J Public Health. 2008;98(6):1043-50.

44. Das M, Chu PL, Santos GM, Scheer S, Vittinghoff E, McFarland $\mathrm{W}$, et al. Decreases in community viral load are accompanied by reductions in new HIV infections in San Francisco. PLoS ONE. 2010;5(6): 11068 .

45. Bailey ZD, Krieger N, Agénor M, Graves J, Linos N, Bassett MT. Structural racism and health inequities in the USA: evidence and interventions. Lancet. 2017;389(10077):1453-63.

46. Nieblas R, Hughes L, Andrews R, Relf M. Reframing and understanding the HIV epidemic in MSM: Masculinity, racism, and homophobia. J Assoc Nurses AIDS Care. 2015;26(5):514-9.

47. Matthews DD, Smith JC, Brown AL, Malebranche DJ. Reconciling epidemiology and social justice in the public health discourse around the sexual networks of black men who have sex with men. Am J Public Health. 2016;106(5):808-14.

48. Herbst JH, Sherba RT, Crepaz N, DeLuca JB, Zohrabyan L, Stall $\mathrm{RD}$, et al. A meta-analytic review of HIV behavioral interventions for reducing sexual risk behavior of men who have sex with men. JAIDS J Acquir Immune Defic Syndr. 2005;39(2):228-41.

49. Maulsby C, Millett G, Lindsey K, Kelley R, Johnson K, Montoya $\mathrm{D}$, et al. A systematic review of HIV interventions for black men who have sex with men (MSM). BMC Public Health. 2013;13(1):625.

50. Baral S, Logie CH, Grosso A, Wirtz AL, Beyrer C. Modified social ecological model: a tool to guide the assessment of the risks and risk contexts of HIV epidemics. BMC Public Health. 2013;13(1):482.

51. Blankenship KM, Smoyer AB, Bray SJ, Mattocks K. Black-white disparities in HIV/AIDS: the role of drug policy and the corrections system. J Health Care Poor Underserved. 2005;16(4 Suppl B): 140 .

52. Fujimoto K, Wang P, Ross MW, Williams ML. Venue-mediated weak ties in multiplex HIV transmission risk networks among drug-using male sex workers and associates. Am J Public Health. 2015;105(6):1128-35.

53. Moher D, Liberati A, Tetzlaff J, Altman DG (2009) Preferred reporting items for systematic reviews and meta-analyses: the PRISMA statement. Ann Internal Med. 2009; 151(4):264-69.

54. Raymond HF, Chen Y-H, Syme SL, Catalano R, Hutson MA, McFarland W. The role of individual and neighborhood factors: HIV acquisition risk among high-risk populations in San Francisco. AIDS Behav. 2014;18(2):346-56.

Publisher's Note Springer Nature remains neutral with regard to jurisdictional claims in published maps and institutional affiliations. 